\title{
Clinical Results of Lumboperitoneal Shunt with a Valve Reservoir Compared with Ventriculoperitoneal Shunt
}

\author{
Joon-Ho Yoon ${ }^{1}$, Ho-Shin Gwak', Ji-Woong Kwon³, Sang-Hoon Shin ${ }^{3}$, Heon Yoo \\ ${ }^{1}$ Department of Neurosurgery, Seoul National University College of Medicine, Seoul, ${ }^{2}$ Department of System Cancer \\ Science, Graduate School of Cancer Science and Policy, National Cancer Center, Goyang, ${ }^{3}$ Neuro-Oncology Clinic, \\ National Cancer Center, Goyang, Republic of Korea
}

Corresponding author:

Ho-Shin Gwak

Department of System Cancer

Science, Graduate School of

Cancer Science and Policy,

National Cancer Center, 323

Ilsan-ro, Ilsandong-gu, Goyang

10408, Republic of Korea

Tel: $+82-31-920-1666$

Fax: +82-31-920-2798

E-mail: nsghs@ncc.re.kr

Received: August 24, 2017

Revised: September 26, 2017

Accepted: October 3, 2017

\begin{abstract}
Objective: Lumboperitoneal (LP) shunt has not been commonly used due to frequent over-drainage and malfunction. We adapted an LP shunt with a valve reservoir or programmable valve for use in patients who needed to avoid cranial surgery. We compared the clinical results of the LP shunt with a ventriculoperitoneal (VP) shunt. Methods: Among 188 shunt installations, we selected 132 patients who received a first-time shunt installation (108 VP shunt and 24 LP shunt) to compare clinical improvement, shunt malfunction, over- and under-drainage, and infection rates along with clinical factors between the two groups of patients. Results: Clinical improvement occurred in 107 (80\%) patients. The rates of clinical improvement were not significantly different between two groups. Malfunction and infection was recorded in 15 (11\%) patients and in 8 (6\%) patients, respectively. The incidence for malfunction and infection were not significantly different between two groups. Over-drainage and under-drainage were recorded in 19 patients, each. The need for valve adjustment was not significantly different between two groups $(p=0.46)$. Shunt revision was performed in 31 (24\%) patients. The proportion of patients received revisions in the LP shunt group tended to be greater (9/24) compared with the VP shunt group $(22 / 108)(p=0.07)$. However, the total revision rates (number of revision/ total shunt) were not significantly different between the two groups $(p=0.74)$. Conclusion: We suggest clinical efficacy of LP shunt with a valve resenoir is compatible with VP shunt, and recommend use of this system in patients with communicating hydrocephalus.
\end{abstract}

Key Words: Cerebrospinal fluid shunts; Lumbar region; Ventriculoperitoneal shunt

\section{INTRODUCTION}

Cerebrospinal fluid (CSF) shunting procedures are widely used to divert disturbed CSF flow in patients with hydrocephalus (HCP). HCP is divided into communicating or non-communicating (obstructive) HCP, based on pathophysiology. While a ventriculoperitoneal (VP) shunt can be used to treat obstructive or communicating HCP, use of a lumboperitoneal (LP) shunt should be restricted to treatment of communicating HCP. Despite this restriction, the LP shunt has advantages over the VP shunt such as avoidance of cranial surgery, less severe complications (e.g., intracranial hemorrhage [ICH]), accessibility for small ventricle HCP, and use for CSF leakage. During the early years of LP shunt use, the conventional LP shunt consisted of a valveless, thin, Silastic tube system (one-piece); the lack of a reservoir to assess shunt function and a valve to adjust the CSF flow were major disadvantages ${ }^{2,4,7)}$. Patients with these LP shunts frequently experienced over-drainage symptoms and obstruc- tion, kinking, or migration ${ }^{2,7)}$. One study comparing LP with VP shunt efficacy found inferior rates of clinical improvement when the LP shunt was used ${ }^{8)}$. After Spetzler et al. ${ }^{14)}$ introduced use of spring-reinforced tubing and a flank-anchored reservoir, the rates of LP shunt complications such as kinking, adhesive arachnoiditis, and migration were greatly reduced. And also, an access to examination of shunt patency became possible ${ }^{10)}$. The LP shunt with a flushing reservoir was actively adopted to divert CSF flow disturbance in patients with a small or slit ventricle (e.g., pseudotumor cerebri [idiopathic intracranial hypertension] $)^{6,11}$. Compared with the VP shunt, these LP shunt systems with a reservoir (two-piece or three-piece) have equal or reduced rates of shunt-related complications. However, problems related to over-drainage (e.g., subdural hematoma and aggravation of Chiari malformation) have been reported ${ }^{1,5)}$. After introduction of the valve reservoir, the rates of over-drainage and associated complications were greatly reduced ${ }^{16)}$. Since the development of a programmable valve, the LP shunt has been widely used for communicating HCP conditions including 
normal pressure HCP (NPH), pseudotumor cerebri, intraventricular hemorrhage (IVH), and subarachnoid hemorrhage ${ }^{3,8,12}$. Recently, we began performing the LP shunt instead of the VP shunt in leptomeningeal carcinomatosis (LMC) patients because the LP shunt is a relatively simple procedure and avoids cranial surgery. At our institution, LP shunts with valve reservoirs are given to patients with LMC, NPH, or CSF leakage with scalp problem, at the surgeon's choice.

There are few studies reporting the clinical results and efficacy of the use of the LP shunt compared with the VP shunt, and no consensus has been reached ${ }^{1,11}$. We analyzed the results for clinical improvement and complications for LP shunt compared with VP shunt use at a single institution.

\section{MATERIALS AND METHODS}

\section{Patient Eligibility}

From December 2001 to May 2017, we performed 206 cases of shunt surgery. We excluded two cases of syrinx-spinal shunt and a case of subduroperitoneal shunt as those cases were different from VP and LP shunts in terms of clinical evaluation results. Among 203 cases of shunt surgery, 15 cases were removal of infected or malfunction shunt devices and extraventricular drainage. An additional 56 cases were shunt surgery revisions (47 VP shunts and 9 LP shunts). After excluding those cases, we evaluated the clinical results for 132 patients who received a first-time shunt installation (108 patients with a VP shunt and 24 patients with an LP shunt) (Table 1).

\section{LP Shunt Installation}

All procedures were performed under general anesthesia with the patient in a lateral position. The proximal incision for spinal subarachnoid catheter insertion and anchoring was approximately $3 \mathrm{~cm}$ in length and made horizontally at the interspinous process L2-3 or L3-4 levels. A Tuohy needle (14gauge) was inserted into the CSF space with or without fluoroscopy guidance. A small diameter proximal catheter was then inserted up to $10 \mathrm{~cm}$ inside the space. Another incision was made on the anterior superior iliac spine or on the end of the $12^{\text {th }}$ rib in obese patients using a semi-circular to hockey-stick shape (Figs. 1, 2). After connecting the proximal and distal catheters to the valve reservoir (Spetzler LP shunt system; Integra NeuroSciences, Plainsboro, NJ, USA) or programmable NSC valve (Starata LP hunt; Medtronic, Goleta, CA, USA), the reservoir was anchored to the periosteum and fascia. The distal catheter was inserted into the periosteum through an incision made at McBurney's point or the periumbilical area.
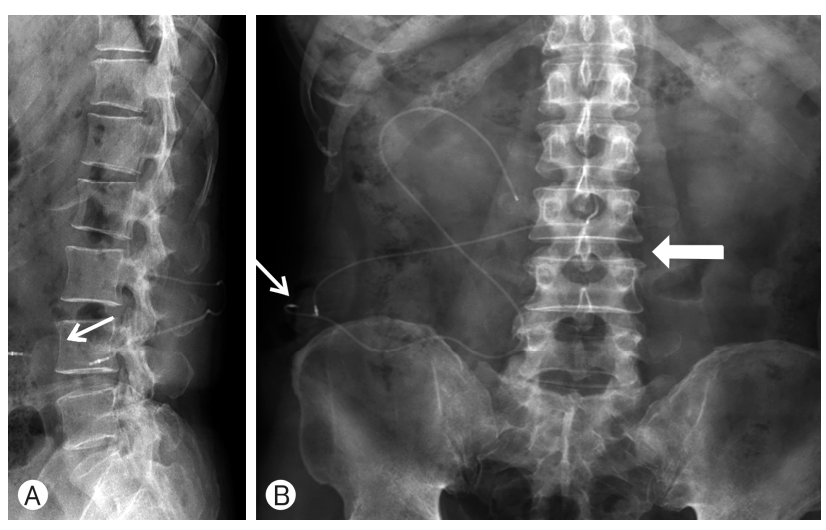

Fig. 1. Lumboperitoneal shunt using the anterior superior iliac spine as the reservoir anchoring point, via an L3-4 puncture. (A) Lateral view, (B) anterior-posterior view roentgenography. Arrows indicate the reservoir location; the white arrow indicates the lumbar puncture level.

Table 1. Characteristics of patients with LMC of non-small-cell lung cancer with or without magnetic resonance imaging finding of LMC

\begin{tabular}{|c|c|c|c|c|}
\hline Characteristics & All patients $(n=132)$ & VP shunt $(n=108)$ & LP shunt $(n=24)$ & $\mathrm{p}$-value \\
\hline Mean age $\pm S D$ & $45.5 \pm 22.7$ & $43.3 \pm 23.7$ & $55.3 \pm 14.5$ & 0.018 \\
\hline Range & $1-90$ & $1-90$ & $18-81$ & \\
\hline Gender & & & & 0.49 \\
\hline Male & $69(52.3 \%)$ & $58(53.7 \%)$ & $11(45.8 \%)$ & \\
\hline Female & $63(47.7 \%)$ & $50(46.3 \%)$ & $13(54.2 \%)$ & \\
\hline Preceding disease & & & & 0.018 \\
\hline Brain tumor & $56(42.4 \%)$ & 52 (48.1\%) & $4(16.7 \%)$ & \\
\hline LMC & 62 (47.0\%) & $46(42.6 \%)$ & $16(66.7 \%)$ & \\
\hline Others* & $14(10.6 \%)$ & $10(9.3 \%)$ & $4(16.7 \%)$ & \\
\hline Opening pressure $\left(\mathrm{cm} \mathrm{H}_{2} \mathrm{O}\right)$ & & & & 0.90 \\
\hline Mean \pm SD & $21.1 \pm 13.1$ & $21.0 \pm 12.2$ & $21.6 \pm 20.8$ & \\
\hline Range & $0-80$ & $0-80$ & $3-60$ & \\
\hline
\end{tabular}

LMC: leptomeningeal carcinomatosis; VP: ventriculoperitoneal; LP: lumboperitoneal; SD: standard deviation.

*Others include cerebrospinal fluid leakage, hemorrhage, idiopathic intracranial hypertension and normal pressure hydrocephalus. 


\section{Evaluated Parameters}

The clinical results were defined based on the patient's subjective statements or the physician's description on the day of discharge after the procedure or at the first outpatient visit after the shunt operation. The results were classified as: (1) normalized - all HCP-related symptoms were resolved; (2) improved - discernible improvement of the symptoms; and (3) no improvement.

The variables used to indicate shunt surgery complications were: (1) the rate of malfunction; (2) development of over-/underdrainage symptoms; (3) CSF infection; (4) the number of patients requiring shunt revision; and (5) the revision rate (accumulated number of shunt revisions/number of total shunt operations). Cases of malfunction were confirmed using a shunt function test or operative findings. Determination of over- or under-drainage was based on clinical findings and follow-up neuroimaging results. The infection cases included clinically suspicious infections without microorganism isolation. Clinical factors such as age, gender, primary disease, and opening pressure were evaluated together to clarify whether there were any significant differences between the VP shunt and LP shunt groups.

\section{Statistical Analysis}

The evaluated scaled parameters were compared using the
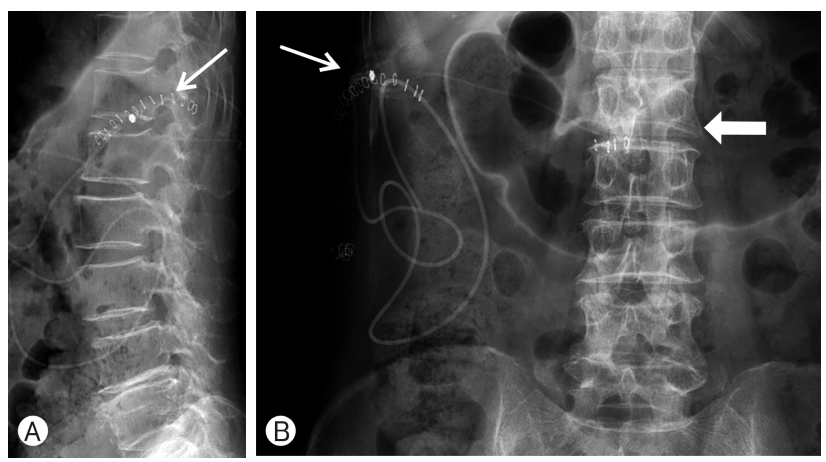

Fig. 2. Lumboperitoneal shunt using the $12^{\text {th }}$ rib as the reservoir anchoring point via an L2-3 puncture. (A) Lateral view, (B) anterior-posterior view roentgenography. Arrows indicate the reservoir location; the white arrow indicates the lumbar puncture level.
Student's t-test. Categorical variables were compared quantitatively between the VP shunt and LP shunt groups using the $\mathrm{x}^{2}$ test or Fisher's exact test. We considered a $\mathrm{p}<0.05$ to indicate a statistically significant difference. All calculations were performed using the R 5.1 version freely available on the web (http://cran.r-project.org/).

\section{RESULTS}

\section{Clinical Characteristics of Patients}

A total of 132 patients received 188 shunt operations during the study period; these operations included shunt revisions. One-hundred eight patients received VP shunt and among 24 patients having LP shunt, 15 patients had fixed pressure valve reservoir and 9 patients had a programmable valve (Table 1). The mean age was 45.5 years (standard deviation $[\mathrm{SD}]=22.7$ ); the age range was 1 to 90 years. The mean age was significantly younger in patients who received a VP shunt compared with patients who received an LP shunt (43.3 years vs. 55.3 years, $\mathrm{p}=0.018$ ). The gender distribution was nearly equal (69 male patients and 63 female patients); this distribution was not significantly different according to the shunt type. At our institution, LMC was the most common disease (62 patients, $47 \%$ ) preceding the HCP; brain tumor (56 patients, 42\%) was the next most frequent disease. There were seven cases of post-hemorrhage $\mathrm{HCP}$, four cases of NPH, two cases of CSF leakage, and one case of idiopathic intracranial hypertension. As expected, a communicating HCP resulting from LMC was the most frequent (16/24, 67\%) preceding disease in LP shunt patients; the proportion was significantly greater compared with VP shunt patients (46/108, 43\%; $\mathrm{p}=0.018)$. The mean opening pressure measured at the time of proximal catheter insertion was $21.1 \mathrm{~cm}$ $\mathrm{H}_{2} \mathrm{O}(\mathrm{SD}=13.1)$ and ranged from 0 to $80 \mathrm{~cm} \mathrm{H}_{2} \mathrm{O}$. These pressures were not significantly different according to the applied shunt type.

\section{Clinical Improvement}

After shunt installation, 56 (42\%) patients stated that all preoperative HCP-related symptoms were 'normalized', and 51 (39\%) patients described their symptoms as 'improved'. The remaining 25 (19\%) patients did not experience any improve-

Table 2. Comparison of clinical improvement between VP shunt and LP shunt

\begin{tabular}{lcccc}
\hline \hline Malfunction & All patients $(\mathrm{n}=132)$ & VP shunt $(\mathrm{n}=108)$ & LP shunt $(\mathrm{n}=24)$ & $\mathrm{p}$-value \\
\hline Normalized & $56(42.4 \%)$ & $50(46.3 \%)$ & $6(25.0 \%)$ & \\
Improved & $51(38.6 \%)$ & $40(37.0 \%)$ & $11(45.8 \%)$ & $0.13^{*}$ \\
Not improved & $25(19.0 \%)$ & $18(16.7 \%)$ & $7(29.2 \%)$ & \\
\hline
\end{tabular}

VP: ventriculoperitoneal; LP: lumboperitoneal.

*Normalized+improved vs. not improved, $p=0.16$; Normalized vs. improved+not improved, $p=0.056$. 
ment in symptoms (Table 2). There was no significant difference of clinical improvement according to the shunt type $(\mathrm{p}=0.13)$. In detail, the difference between the proportions of patients who experienced improvement (normalized + improved) was not statistically significant between the VP and LP shunt groups $(\mathrm{p}=0.16)$. However, there was a tendency for normalization (normalized vs. improved + not improved) to occur in a greater proportion of patients in the VP shunt group $(56 / 108,52 \%)$, compared with the LP shunt group patients $(6 / 24,25 \%$; $\mathrm{p}=0.056)$ (Table 2).

\section{Shunt Malfunction}

Shunt malfunction occurred in $15(11 \%)$ patients. Three patients experienced a proximal malfunction and 10 patients experienced a distal malfunction; the remaining 2 patients experienced total malfunction. The shunt malfunction incidence was not significantly different between the VP shunt and LP shunt groups $(\mathrm{p}=0.37$ ) (Table 3). Distal malfunction occurred in the all four LP patients who experienced shunt malfunction.

\section{ICH}

Two patients of VP shunt group had IVH postoperatively. One patient with anti-coagulant therapy for heart disease had IVH but spontaneously resolved without shunt malfunction. The other patient was found altered mentality on postoperative 10 days, and brain CT revealed $\mathrm{IVH}$. She needed an extraventricular drainage. There was no ICH in patients of LP shunt group.

\section{Shunt Infection}

Infection of the shunt device occurred in 8 patients ( 4 patients from each group) (Table 3). This apparently greater proportion of infections in LP shunt patients was statistically significant $(\mathrm{p}=0.016)$. In detail, 3 out of 4 shunt infections in LP patients occurred during weekly or twice per week intraventricular chemotherapy via the Ommaya reservoir. This chemotherapy system was not applied for VP shunt patients.

\section{Need for Valve Adjustment}

Documented valve adjustment was required in 38 patients; 33 patients had a VP shunt and 5 patients had an LP shunt. The need for valve adjustment was not significantly different between the two shunt types $(\mathrm{p}=0.34)$ (Table 3$)$. Over-drainage occurred in 19 patients and under-drainage occurred in 19 patients. Over-drainage occurred more frequently than underdrainage in patients with an LP shunt (4 patients vs. 1 patient). Over-drainage and under-drainage occurred in 15 patients and 18 patients, respectively, in patients with a VP shunt. This propensity for over-drainage in LP shunt patients was not statistically significant $(\mathrm{p}=0.15)$.

\section{Shunt Revision}

Twenty-two (20\%) VP shunt and nine (38\%) LP shunt patients underwent shunt revision due to malfunction or infection

Table 3. Comparison of malfunction and infection rate between VP shunt and LP shunt

\begin{tabular}{|c|c|c|c|c|}
\hline Malfunction & All patients $(n=132)$ & VP shunt $(n=108)$ & $L P$ shunt $(n=24)$ & $p$-value \\
\hline Malfunction & & & & $0.37^{*}$ \\
\hline Yes & 15 (11.4\%) & 11 (10.2\%) & $4(16.7 \%)$ & \\
\hline No & 117 (88.6\%) & 97 (89.8\%) & 20 (83.3\%) & \\
\hline Need to adjustment & & & & $0.34^{*}$ \\
\hline Yes & 38 (28.8\%) & 33 (30.6\%) & 5 (20.8\%) & \\
\hline No & 94 (71.2\%) & 75 (69.4\%) & 19 (79.2\%) & \\
\hline Hemorrhage & & & & N/A \\
\hline Yes & $2(1.5 \%)$ & $2(1.9 \%)$ & $0(0.0 \%)$ & \\
\hline No & 130 (98.5\%) & 106 (98.1\%) & 24 (100.0\%) & \\
\hline Infection & & & & $0.016^{*}$ \\
\hline Yes & $8(6.1 \%)$ & $4(3.7 \%)$ & $4(16.7 \%)$ & \\
\hline No & 124 (93.9\%) & 104 (96.3\%) & 20 (83.3\%) & \\
\hline Revision & & & & 0.073 \\
\hline Yes & 31 (23.5\%) & $22(20.4 \%)$ & 9 (37.5\%) & \\
\hline No & 101 (76.5\%) & 86 (79.6\%) & 15 (62.5\%) & \\
\hline Revision rate ${ }^{* *}$ & $57 / 188$ & $47 / 155$ & 10/33 & 0.99 \\
\hline
\end{tabular}

VP: ventriculoperitoneal; LP: lumboperitoneal; N/A: not available.

*Fisher's exact test.

**Number of shunt revision/total shunt operation (see detail in Methods). 
(Table 3). The LP shunt patient group had a tendency towards a greater proportion of shunt revision, compared with the VP shunt group $(\mathrm{p}=0.073)$. As 47 of $155 \mathrm{VP}$ shunt operations were revisions, and 10 of $33 \mathrm{LP}$ shunts were revision surgery, there was no significant difference in terms of total revision rate (number of revision/ total shunt operation) between patients with a VP shunt and patients with an LP shunt $(\mathrm{p}=0.99)$. Among 9 patients who underwent LP shunt revision, 5 had a fixed pressure valve and 4 had a programmable valve. The difference in revision rate was not statistically significant according to valve type $(\mathrm{p}=0.68)$.

Sixty-two patients had either VP or LP shunts due to LMC, and were followed at a median of 2.2 months $(95 \%$ confidence interval=2.5-6.0) and 14 patients underwent shunt revisions. Among 4 catheter obstructions documented, one was proved to be foreign body debris on pathological examination of the catheter. In the other 3, pathological examination was not submitted. There was no valve obstruction from floating cancer cells among shunt revision cases.

\section{DISCUSSION}

This study was limited by its retrospective design and use of heterogeneous patient groups in terms of primary disease and shunt devices used. However, our results did indicate the efficacy of the LP shunt with a valve reservoir for treatment of communicating HCP in patients with brain tumor and LMC, compared with the use of the VP shunt. No previously published studies have included findings for this comparison.

\section{Clinical Indications and Effectiveness of LP Shunt}

An indication for LP shunt use is limited to use of communicating HCP because the LP shunt does not divert CSF proximal to the obstruction. According to a nationally-based report for the USA population, 5,574 shunt procedures were performed in 2000; the percentages of non-communicating HCP and communicating HCP were $16.6 \%$ and $13.2 \%$, respectively ${ }^{13)}$. Thus, we can assume that application of the LP shunt is as common as that of VP shunt. Studies of the clinical results of LP shunt use found that that the most common preceding diseases are hemorrhage, infection, pseudotumor cerebri (idiopathic intracranial hypertension), and CSF fistulae with small ventricle sizes ${ }^{2,16)}$.

The reported symptom control rate varies according to the LP shunt system used. Comparison of the clinical effectiveness of the LP and VP shunts in terms of the control rate would be useful if all cases included communicating HCP and possibly one preceding disease. Kang ${ }^{8)}$ studied the efficacies of LP vs. VP shunting for treatment of chronic HCP following aneurysmal subarachnoid hemorrhage. All LP shunts they used were a onepiece tube type without a reservoir. Therefore, clinical improvement was significantly less in the patients who received the
LP shunt, compared with the patients who received the VP shunt $(50 \%$ vs. 91\%; $\mathrm{p}<0.005)$. Menger et al. ${ }^{11)}$ performed a nationwide analysis of the clinical results of LP vs. VP shunt use for 4,480 patients with idiopathic intracranial hypertension. In terms of medical costs, lengths of hospital stays, and revision rates (7.0\% vs. 3.9\%; $\mathrm{p}<0.0001)$, they questioned selection of the LP shunt as the first-line treatment for idiopathic intracranial hypertension. However, they did not clarify which LP shunt system was used for 1,754 patients who received an LP shunt as the first-time shunt placement. Our study findings indicated that although the clinical improvement rate (normalized plus improved) was not significantly different between the two shunt groups, there was a tendency for greater HCP symptom normalization in VP shunt, compared with LP shunt, patients. Resolution of all pre-shunt symptoms is very difficult in LMC patients; they generally experience various neurological deficits. Thus, we suggest that the observed tendency was a consequence of a greater proportion of LMC patients in the LP shunt group (67\%) than in the VP shunt group (43\%).

\section{Complications Following LP Shunt}

VP shunt placement is more likely to be than LP shunt placement, even in cases of communicating HCP. However, severe complications such as ICH can result from VP shunt placement. Our results indicated that no shunt-associated hemorrhage occurred in patients with an LP shunt; ICH did occur in VP shunt patients.

LP shunt complication rates range from 20.5\% to 85.7\%; rates vary between studies and depend on the LP shunt system used $^{1,5,10,17)}$. Migration was a frequent cause of revision for onepiece tube type LP shunt ${ }^{6,9)}$, but was rarely reported after adoption of the reservoir/valve system ${ }^{1,3,10,15)}$. Aoki ${ }^{11}$ used a twopiece LP shunt system with a flushing reservoir. They found significantly fewer complications in patients who received the LP shunt (52/207), compared with patients who received a VP shunt $(62 / 120),(\mathrm{p}<0.0001)$. The over-drainage symptoms that occur after LP shunt placement have long been a major disadvantage compared with the VP shunt; some patients develop subdural fluid collection, chronic subdural hemorrhage, or iatrogenic Chiari malformation ${ }^{1,5,9,10,17)}$. Wang et al. ${ }^{16)}$ suggested that the LP shunt valve reservoir system has an important role in prevention of over-drainage. In their study population, 11 out of 74 patients with an LP shunt experienced over-drainage symptoms. Seven of these patients were from a group of 21 patients who received valveless LP shunts; 4 patients were from a group of 46 patients whose LP shunt included a valve system $(p=0.01)$. A recent study of use of an LP shunt with a programmable valve found none of the above-mentioned over- drainage symptoms among 20 patients, except for a case of spontaneous valve readjustment that was resolved using a valve reset ${ }^{15)}$.

Our study revealed that 9 out of 24 (38\%) patients with a LP shunt needed revision; all patients had a fixed pressure 
type or programmable valve shunt. There was a tendency towards a higher revision probability in LP compared with VP shunt patients. However, the proportion of patients for whom infection was the cause for revision was greater in the LP shunt (4/9, 44\%) vs. the VP shunt (4/22, 18\%), group. Infection was less likely the result of shunt malfunction but was more likely in proportion to the need for CSF sampling or chemotherapeutic agent injection in LMC patients. As mentioned, 3 out of 4 infections from LP shunt occurred in patients who had received intraventricular chemotherapy via Ommaya reservoir.

\section{Future Directions}

Nadkarni et al. ${ }^{12)}$ reported the efficacy of an LP shunt with a programmable valve for patients with pseudotumor cerebri, with use of a concurrent ventricular access device (Rickham reservoir, Codman corp.) to monitor intracranial pressure after LP shunt function. Zhang et al.' ${ }^{18)}$ review found that the LP shunt is more suitable for use for LMC compared with the VP shunt; its advantages include a relatively simple and less invasive procedure. They suggested use of the LP shunt for LMC patients with a normal ventricle size, in combination with intraventricular Ommaya reservoir, in a context similar to Nadkarni et al. ${ }^{12)}$. In line with their expectation, we used intraventricular Ommaya reservoirs for the entry of CSF chemotherapy for 6 LMC patients with an LP shunt. This approach was not possible in patients with a VP shunt. Future studies could be used to examine the effectiveness of controlling HCP via an LP shunt while continuing intraventricular chemotherapy via Ommaya reservoir in patients with LMC.

\section{CONCLUSION}

In this study, we evaluated that use of an LP shunt with a fixed pressure or programmable valve system had performance comparable to use of a VP shunt. Although there was a greater tendency for revision in patients with an LP shunt after the first installation, the total revision number was similar to the VP shunt. No severe complications associated with ICH occurred in LP shunt patients. Thus, we suggest an LP shunt system with valve reservoir should be recommended for treatment of communicating HCP, especially for patients with a small ventricle or LMC.

\section{CONFLICTS OF INTEREST}

No potential conflict of interest relevant to this article was reported.

\section{ACKNOWLDGMENTS}

This work was supported by grants from the National Cancer Center, Korea (NCC-1511000-3 and NCC-1710871-1), and Ministry of Health and Welfare, Research fund (1731340-1).

\section{REFERENCES}

1. Aoki N: Lumboperitoneal shunt: clinical applications, complications, and comparison with ventriculoperitoneal shunt. Neurosurgery 26:998-1003, 1990

2. Aschoff A, Kremer P, Hashemi B, Kunze S: The scientific history of hydrocephalus and its treatment. Neurosurg Rev 22:67-93, 1999

3. Bloch O, McDermott MW: Lumboperitoneal shunts for the treatment of normal pressure hydrocephalus. J Clin Neurosci 19: 1107-1111, 2012

4. Chiewvit S, Nuntaaree S, Kanchaanapiboon P, Chiewvit P: Assessment lumboperitoneal or ventriculoperitoneal shunt patency by radionuclide technique: a review experience cases. World J Nucl Med 13:75-84, 2014

5. Duthel R, Nuti C, Motuo-Fotso MJ, Beauchesne P, Brunon J: Complications of lumboperitoneal shunts. A retrospective study of a series of 195 patients (214 procedures). Neurochirurgie 42: 83-89, 1996

6. Eggenberger ER, Miller NR, Vitale S: Lumboperitoneal shunt for the treatment of pseudotumor cerebri. Neurology 46:15241530, 1996

7. Eisenberg HM, Davidson RI, Shillito J, Jr.: Lumboperitoneal shunts. Review of 34 cases. J Neurosurg 35:427-431, 1971

8. Kang S: Efficacy of lumbo-peritoneal versus ventriculo-peritoneal shunting for management of chronic hydrocephalus following aneurysmal subarachnoid haemorrhage. Acta Neurochir (Wien) 142:45-49, 2000

9. Karabatsou K, Quigley G, Buxton N, Foy P, Mallucci C: Lumboperitoneal shunts: are the complications acceptable? Acta Neurochir (Wien) 146:1193-1197, 2004

10. Kuwana N, Kuwabara T: Lumbar subarachnoid-peritoneal shunt-follow-up study on 158 cases. Neurol Med Chir (Tokyo) 24: 485-489, 1984

11. Menger RP, Connor DE, Jr., Thakur JD, Sonig A, Smith E, Guthikonda B, et al.: A comparison of lumboperitoneal and ventriculoperitoneal shunting for idiopathic intracranial hypertension: an analysis of economic impact and complications using the Nationwide Inpatient Sample. Neurosurg Focus 37:E4, 2014

12. Nadkarni TD, Rekate HL, Wallace D: Concurrent use of a lumboperitoneal shunt with programmable valve and ventricular access device in the treatment of pseudotumor cerebri: review of 40 cases. J Neurosurg Pediatr 2:19-24, 2008

13. Patwardhan RV, Nanda A: Implanted ventricular shunts in the United States: the billion-dollar-a-year cost of hydrocephalus treatment. Neurosurgery 56:139-144, 2005

14. Spetzler RF, Wilson CB, Grollmus JM: Percutaneous lumboperitoneal shunt. Technical note. J Neurosurg 43:770-773, 1975

15. Toma AK, Dherijha M, Kitchen ND, Watkins LD: Use of lumboperitoneal shunts with the Strata NSC valve: a single-center experience. J Neurosurg 113:1304-1308, 2010

16. Wang VY, Barbaro NM, Lawton MT, Pitts L, Kunwar S, Parsa AT, et al.: Complications of lumboperitoneal shunts. Neurosurgery 60:1045-1048, 2007

17. Yadav YR, Pande S, Raina VK, Singh M: Lumboperitoneal shunts: review of 409 cases. Neurol India 52:188-190, 2004

18. Zhang XH, Wang XG, Piao YZ, Wang P, Li P, Li WL: Lumboperitoneal shunt for the treatment of leptomeningeal metastasis. Med Hypotheses 84:506-508, 2015 